\title{
Regular periods of abdominal contractions recorded from larvae of the bumblebee, Bombus terrestris (Hymenoptera: Apidae)
}

\author{
Marika MÄND ${ }^{1}$, Aare KUUSIK ${ }^{1}$, AnTs-JohanNes MARTIN ${ }^{1}$, INGRid H. WILLIAMS ${ }^{2}$, ANNe LUIK ${ }^{1}$, Reet KARISE ${ }^{1}$, \\ LUULE METSPALU ${ }^{1}$ and KÜLLI HIIESAAR ${ }^{1}$ \\ ${ }^{1}$ Institute of Agricultural and Environmental Sciences, Estonian University of Life Sciences, Kreutzwaldi 64, EE-51014 Tartu, \\ Estonia; e-mail: marika.mand@ut.ee \\ ${ }^{2}$ Rothamsted Research, Harpenden, Hertfordshire, AL5 2JQ, UK; e-mail: ingrid.williams@bbsrc.ac.uk
}

Keywords. Bombus terrestris, larvae, active ventilation, extracardiac pulsations, heartbeat, respiratory responses

\begin{abstract}
Using an opto-cardiograph combined with an infrared gas analyzer regular bouts of abdominal contractions were recorded from last instar larva of Bombus terrestris. The rate of $\mathrm{CO}_{2}$ release was about $0.7 \mathrm{ml} \mathrm{g}^{-1} \mathrm{~h}^{-1}$. The bouts of contractions were of two types: weak extracardiac pulsations and vigorous pumping. The frequencies of pulsations and pumping were $25-35$ per min and 8-12 per min, respectively. Bouts of extracardiac pulsations and abdominal pumping were independent of each other and sometimes overlapped. Cardiac contractions (heartbeats) were continuous (57-63 pulses/min). This study suggests that the periodically occurring abdominal contractions play an essential role in respiration and/or in haemolymph circulation in larvae of $B$. terrestris.
\end{abstract}

\section{INTRODUCTION}

In adult and pupal stage of many insects, visible abdominal contractions are known to actively ventilate the tracheal system (Buck, 1962; Miller, 1974, 1981; Mill, 1985, for reviews). These vigorous abdominal contractions are known as abdominal pumping.

Sláma (1976) described a repeated series of pulsations regulated by the autonomic nervous system (coelopulse), resulting in changes in hydrostatic pressure within the insect body, which he termed extracardiac haemocoelic pulsations. The pulsations are often microscopic invisible movements of some elastic body segments. According to Sláma (2000), "the pulsations are produced by coordinated rhythmic contractions of intersegmental (dorsoventral in certain species) muscles". These extracardiac pulsations have been described in various developmental stages in a number of insect species. These pulsations are controlled by the autonomic nervous system (coelopulse), which regulates important physiological functions including gaseous exchange through the spiracles and haemolymph circulation (Sláma 1999, for reviews).

In many insects, discontinuous gas exchange cycles or DGCs are associated with active ventilatory movements of the abdomen, during which emission of carbon dioxide is discontinuous (Kestler, 1985; Sláma, 1988; Lighton, 1994, 1996, for reviews).

Although active ventilation has been extensively studied in the pupal and adult stages of insects, little is known about it in the larval stages of terrestrial holometabolous insects. Gas exchange in soft-bodied larvae was thought to be by diffusion alone and not by active ventilation (Krogh, 1920, Wasserthal, 1996). However, ventilatory movements can be very weak and therefore may have been overlooked. Special techniques are now available to study such weak movements. Extracardiac pulsations have been recorded from the tip of the abdomen using an electronic transducer (Sláma, 1984), or by means of an infrared cardiograph referred to as an optocardiograph (Sláma, 2003; Kuusik et al., 2002). Extracardiac pulsations have been recorded also using an electrolytic respirometer-actograph (Kuusik et al., 1994, 1996).

Abdominal contractions have been recorded in the latespinning larvae of Galleria mellonella (Sláma, 1984). The last instar larvae of $G$. mellonella in the wandering stage showed almost continuous rhythmic movements with short pauses, when immobile (Kuusik et al., 1991). Sláma (1999) recorded in prepupae of Cossus cossus continuous radial contractions of the larval somatic muscular sheath with a periodicity of $13-15$ contractions per minute. He demonstrated that simple passive diffusion alone could not meet the respiratory requirements of the larva; active ventilation is also needed. In Drosophila melanogaster, the extracardiac contractions produced by larval somatic muscles or oral armature are very strong (Sláma \& Farkaš, 2005).

The aim of the present study was to investigate the rhythmic abdominal movements and their respiratory responses in mature larvae of Bombus terrestris, using non-invasive methods that allowed recording of abdominal movements and respiration simultaneously without the removal of the larva from its cocoon.

\section{MATERIAL AND METHODS}

\section{Insects}

A commercial colony (Natupol hives) of the bumblebee $B$. terrestris was purchased from Koppert Biological Systems B.V. (the Netherlands). The colony was kept in a nest box in the laboratory at a temperature of $22-25^{\circ} \mathrm{C}$. The temperature in the nest box around the brood was $28-30^{\circ} \mathrm{C}$ and the air humidity was $70-80 \%$ RH. All manipulations of the colony were carried out under red light. The colony was supplied with unlimited amounts of water, sugar or honey solution and fresh pollen col- 


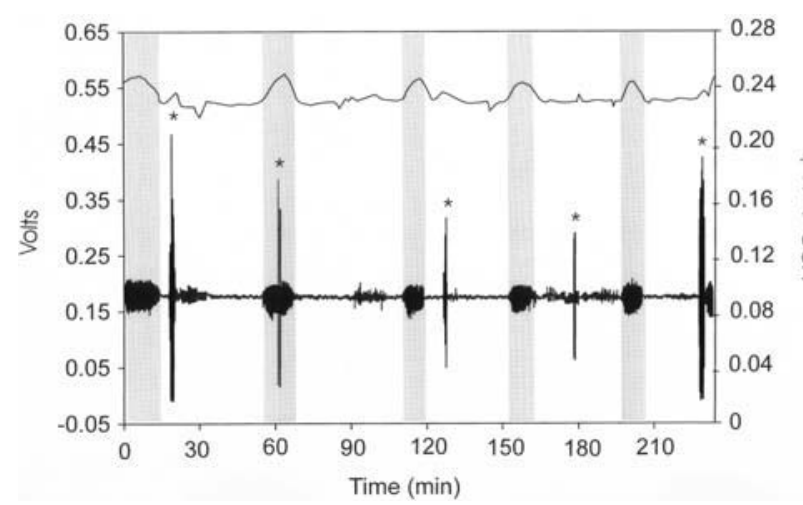

Fig. 1. The lower trace (Volts) is a typical opto-cardiographic recording of the periodically occurring extracardiac abdominal pulsations (grey bars) and abdominal pumping (asterisks) in a larva $(415 \mathrm{mg})$ of Bombus terrestris 2 days before pupal ecdysis. The upper trace is a simultaneous recording of an infrared gas analyser $\left(\mathrm{VCO}_{2}\right)$, demonstrating the respiratory responses to the bouts of abdominal pulsations; note that abdominal pumping results in minor spikes on the recording trace.

lected from honeybee colonies. The larvae were tested soon (4-5 h) after the brood cells were covered. A total of ten mature larvae (390-460 mg) were tested. Neither the colony, nor its larvae were parasitized or infected by disease.

\section{Flow-through respirometry}

An infrared gas analyser (IRGA, Infralyt-4, VEB, Junkalor, Dessau), adapted for entomological research, was used to record the bouts of abdominal contractions via the $\mathrm{CO}_{2}$ signals. IRGA was calibrated at different flow rates by means of calibration gases (Trägergase, VEB, Junkalor, Dessau), with gas injection. Air flow rate was 3.61 per $\mathrm{h}$. The rate of carbon dioxide release was measured $\left(\mathrm{VCO}_{2} \mathrm{ml} \mathrm{h}^{-1}\right)$. All measurements were made at $25^{\circ} \mathrm{C}$. The humidity $(\% \mathrm{RH})$ and temperature inside the insect chamber were recorded on the PC monitor using the Humidity and Temperature Display Instrument for digital HygroClip probes (HygroPalm, Rotronic Company) referred to as a hygrometer. A humidity of about $70 \% \mathrm{RH}$ was maintained by means of moistened filter paper strips inside the chamber.

\section{Opto-cardiography}

The IRGA was combined with an infrared (IR) cardiograph for insects, which we refer to as the opto-cardiograph. This recorded not only cardiac (heart) pulses, but also all extracardiac abdominal muscular contractions, including ventilatory contractions. An IR-emitting diode was placed on one side of the chamber near the ventral side of the abdomen, while an IRsensitive diode was placed on the opposite side of the chamber (see Metspalu et al., 2001, 2002; Kuusik et al., 2002). The light from the IR-diode was modulated by the contractions of the heart and skeletal muscles. The level of output voltage reflected the vigour of the muscular contractions of the insect (see Hetz et al., 1999). Contractions of the abdomen were represented by downward spikes on the recordings, while muscular relaxations were directed upward. To avoid confusion between extracardiac pulsations and heartbeats, the IR sensor was placed on the larval body side of the respiratory chamber. When the IR sensor was placed upon the dorsal side of the larva then heartbeats and other body movements were recorded in parallel.

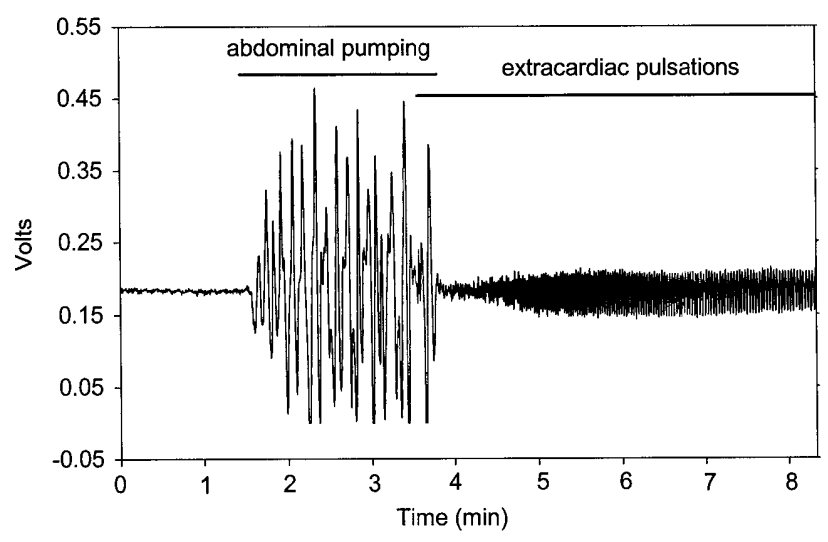

Fig. 2. Detail of the lower trace in Fig. 1 showing the different frequencies and amplitudes of the contractions during a bout of extracardiac abdominal pulsations and abdominal pumping in a larva of Bombus terrestris.

\section{Data acquisition and statistics}

Computerized data acquisition and analysis were performed using DAS 1401 A/D hardware (Keithley, Metrabyte, USA) with a $10 \mathrm{~Hz}$ sampling rate. The two bipolar channels allowed the recording of two events simultaneously.

Tests were performed using the statistics package StatSoft ver. 7, Inc./USA. Values are shown as means \pm standard deviations. Statistical comparisons were performed with nested ANOVA followed by multiple comparison procedures (Tukey test). Probabilities of $P<0.05$ were considered significant.

\section{RESULTS}

The last instar larvae of B. terrestris, 2-3 days before pupal ecdysis, displayed bouts of two types of extracardiac abdominal contractions, here referred to as pulsations and pumping (Fig. 1). Bouts of abdominal pulsations were either short or long. In most individuals $(n=7)$ they were short, lasting 4-6 min, and consisting of 25-30 contractions of almost uniform frequency (30-40 strokes/min). However, in some individuals $(\mathrm{n}=3)$ these bouts were longer, lasting 14-20 min with 60-80 contractions. These longer bouts started with contractions of high frequency (43-53 per min), followed by contractions of a lesser frequency (30-40 per min) and ended with contractions of a lower frequency (25-30 per min) (Fig. 2).

The respiratory responses to the bouts of abdominal pulsations were recorded on the IRGA as a small rise (about 5\%) in carbon dioxide release (peak) (Fig. 1). The mean rate of $\mathrm{CO}_{2}$ release was $0.75 \pm 0.11 \mathrm{ml} \mathrm{g}^{-1} \mathrm{~h}^{-1}(\mathrm{n}=$ 10 individuals).

The bouts of abdominal pumping were more vigorous and slower (8-12 strokes/min) than the bouts of pulsations. They were also less regular, in terms of the amplitude of the contractions and the intervals between the bouts (Fig. 1).

The bouts of pulsations and pumping occurred independently of each other and sometimes overlapped (Figs $3-4)$. The amplitude of the pumping contractions was $2-8$ times larger than that of the pulsation contractions.

The bouts of abdominal pumping produced far smaller peaks on the recording trace than those caused by the 


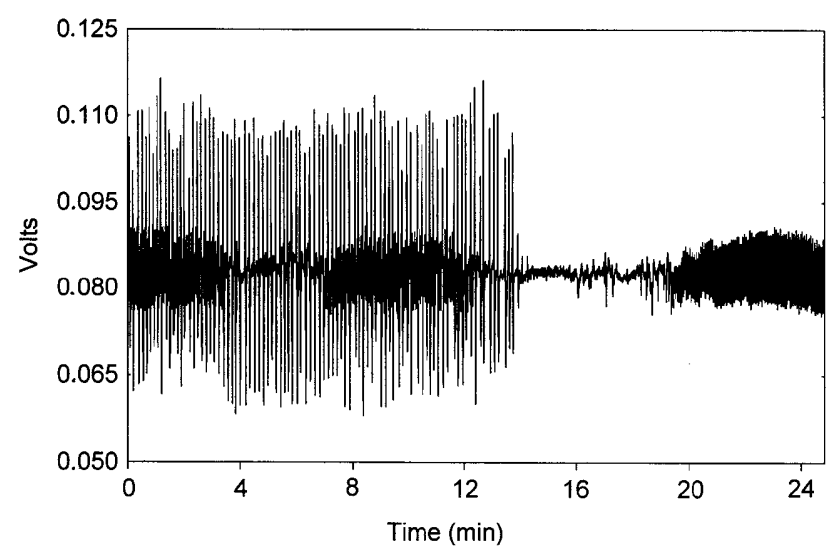

Fig. 3. An opto-cardiographic recording of a larva of Bombus terrestris showing three bouts of extracardiac pulsations. The first two bouts of pulsations (smaller spikes) are overlapped by a bout of abdominal pumping (larger spikes).

bouts of extracardiac pulsations despite the larger amplitude of their strokes (Figs 1-2). Between the peaks caused by the bouts of pulsations, peaks due to decreasing $\mathrm{CO}_{2}$ emission were recorded (Fig. 1); however, the origin of these downward peaks remains unknown.

The heartbeats of the larvae were continuous, with a frequency of about $1 \mathrm{~Hz}\left(58-62\right.$ pulses per min) at $25^{\circ} \mathrm{C}$. On the opto-cardiographic recording trace, the amplitude of heartbeats was at least 4 times shorter than that of the abdominal pulsations (Fig. 4).

Heartbeats, extracardiac pulsations and abdominal pumping were also clearly distinguishable due to their different frequencies: $60.2 \pm 1.6,26.9 \pm 4.1$ and $9.8 \pm 1.7$ strokes per min, respectively (nested ANOVA, $\mathrm{F}_{2,81}=$ 726.1; $P<0.001$; Tukey test, $P<0.05$ ).

\section{DISCUSSION}

This study clearly demonstrated that last instar larvae of the bumblebee, B. terrestris, display regular periods of extracardiac abdominal contractions. Two types of contractions were found: weak movements of the abdominal segments not discernible to the naked eye, the extracardiac pulsations, and visually observable vigorous abdominal pumping. The bouts of abdominal pumping were shorter than those of the pulsations and the frequency of pumping contractions was at least five times slower than that of the pulsations. Sometimes the abdominal pumping and extracardiac pulsations occurred at the same time, indicating that the difference between the two types of contractions is not purely quantitative.

Respiration by the larvae was continuous and the small peaks on the IRGA recording trace were not discontinuous gas exchange cycles (DGCs). In real DGC, the $\mathrm{CO}_{2}$ level falls to zero or close to zero between successive bursts of $\mathrm{CO}_{2}$ i.e. during the interburst period. In the current study, only about $5 \%$ more $\mathrm{CO}_{2}$ was released during the bouts of abdominal pulsations than between the bouts.

Previous studies have shown that extracardiac pulsations are responsible for tracheal ventilation, being directly associated with mechanical emission of gas from

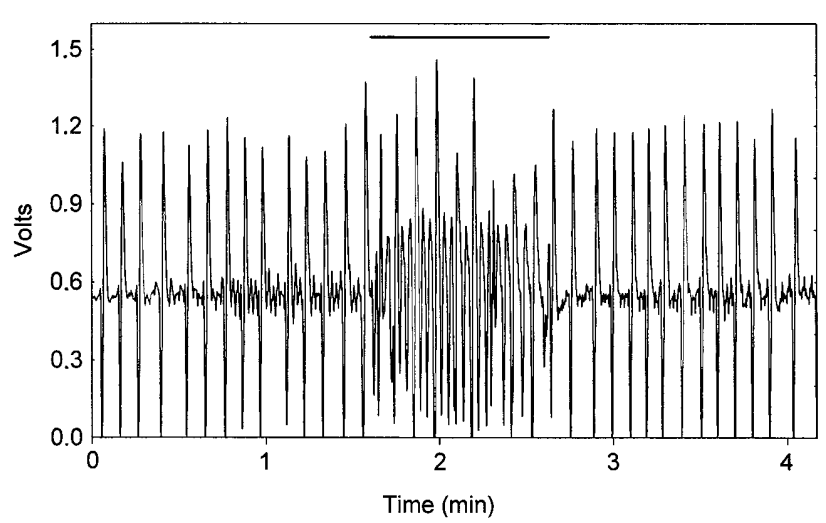

Fig. 4. An opto-cardiographic recording of a larva of Bombus terrestris showing three different types of contractions occurring simultaneously: one bout of extracardiac pulsations (the central medium size spikes indicated by the horizontal line), abdominal pumping movements (large spikes) and cardiac pulsations, i.e. heartbeats (small spikes), visible between the spikes due to pumping movements.

certain spiracles (Sláma, 1999; Sláma \& Neven, 2001). In the current study the spiracular movements were not studied and the exact role of the periodically occurring abdominal contractions in gas exchange remained unclear. The respiratory responses to abdominal pumping were essentially weaker than those to the extracardiac pulsations, which were made with much higher frequency than pumping. Obviously, the spikes on the IRGA recording were at least partly due to the metabolic cost of the muscular activity.

Periodic active ventilation was previously recorded in early pupae of $B$. terrestris, while in old pupae and adults the emission of $\mathrm{CO}_{2}$ is discontinuous (DGC) and always accompanied by active ventilation (Kuusik et al., 2002; Mänd et al., 2005). By comparison, the abdominal contractions in the pupal stage of $B$. terrestris are not as uniform and regular as those in the larval stage. Thus, in $B$. terrestris the patterns of active ventilation in the larvae are similar to those found in the pupae. By contrast, continuous extracardiac pulsations occur in the larvae of $G$. mellonella and C. cossus (Sláma, 1984, 1999). The patterns of active ventilation in larvae of $B$. terrestris are similar to the periodically occurring ventilation movements in pupae.

In the larvae of $B$. terrestris abdominal pulsations, pumping and heartbeats are easily distinguishable by their different frequencies and relative amplitudes. In addition, ventilatory movements occur in bouts and the heartbeats are continuous in the larvae of other holometabolous insects (Wasserthal, 1996).

According to Sláma (1984, 1988, 1999), abdominal pulsations occur in all postembryonic developmental stages of insects, including the larval stage. The respiratory pattern found in the larvae of $B$. terrestris in this study supports this view. 


\section{CONCLUSION}

Active body movements were recorded in the last instar larvae of B. terrestris, 2-3 days before pupal ecdysis. These consisted of two different forms of extracardiac abdominal movement: regular and uniform abdominal pulsations and less regular abdominal pumping. These movements were not continuous but occurred in bouts. The two forms of abdominal contractions appeared to be fully independent of each other and thus regulated independently by nerve centres. The respiratory responses of abdominal pulsations were represented as discrete peaks on the IRGA recording, whereas the periods of pumping were scarcely noticeable on the same recording.

These results suggest that obligatory bouts of abdominal contractions, pulsations and pumping, are involved in respiration and/or circulation in the larvae of $B$. terrestris. There is need of further investigations to elucidate the function of abdominal pulsations and pumping in the larvae of this species.

ACKNOWLEDGEMENTS. This work was supported by Grants from the Estonian Science Foundation, No. 5737, No. 5736, No. 5330 and No. 6722. Rothamsted Research is grant-aided by the Biotechnology and Biological Sciences Research Council, UK.

\section{REFERENCES}

BucK J. 1962: Some physical aspects of insect respiration. Annu. Rev. Entomol. 7: 27-56.

Hetz S.K., Psota E. \& Wasserthal L.T. 1999: Roles of aorta, ostia and tracheae in heartbeat and respiratory gas exchange in pupae of Troides rhadamantus Staudinger 1888 and Ornithoptera priamus L. (Lepidoptera, Papilionidae). Int. J. Insect Morphol. Embryol. 28: 131-144.

Kestlen P. 1985: Respiration and respiratory water loss. In Hoffmann K.H. (ed.): Environmental Physiology and Biochemistry of Insects. Springer, Berlin, pp. 137-183.

Krogh A. 1920: Studien über Tracheenrespiration. II. Über Gasdiffusion in den Tracheen. Pflügers Arch. Ges. Physiol. 179: 95-120.

KuUsiK A., Hiiesaar K., Metspalu L. \& Tartes U. 1991: Gas exchange rhythms of Galleria mellonella L. (Lepidoptera, Pyralidae). Proc. Eston. Acad. Sci. 40: 145-156.

Kuusik A., Tartes U., Harak M., Hilesaar K. \& Metspalu L. 1994: Developmental changes during metamorphosis in Tenebrio molitor (Coleoptera: Tenebrionidae) studied by calorimetric thermography. Eur. J. Entomol. 91: 297-305.

KuUsik A., Harak M., Hilesaar K., Metspalu L. \& Tartes U. 1996: Different types of external gas exchange found in pupae of greater wax moth Galleria mellonella. Eur. J. Entomol. 93: 23-35.

Kuusik A., Martin A.-J., Mänd M., Hitesaar K., Metspalu L. \& TARTES U. 2002: Interrelations of gas exchange cycles, body movements and heartbeats in the foragers of bumblebee Bombus terrestris (Hymenoptera: Apidae) at low temperatures. Eur. J. Entomol. 99: 209-214.

Lighton J.R.B. 1994: Discontinuous ventilation in terrestial insects. Physiol. Zool. 67: 142-162.

Lighton J.R.B. 1996: Discontinuous gas exchange in insects. Annu. Rev. Entomol. 41: 309-324.

Mänd M., KuUsik A., Martin A.-J, Williams I.H., Luik A., Karise R., Metspalu L. \& HiIesaar K. 2005: Discontinuous gas exchange cycles and active ventilation in pupae of the bumblebee Bombus terrestris. Apidologie 36: 561-570.

Metspalu L., Kuusik A., Hiiesaar K., Jõudu J., Tartes U. \& HARAK M. 2001: Simultaneous recording of gas exchange cyclicity, body movements and heartbeats in pupae of Leptinotarsa decemlineata Say made by infrared optical method. Norw. J. Entomol. 48: 91-96.

Metspalu L., KuusiK A., Himesaar K. \& Tartes U. 2002: Tonic immobility in adult Colorado Potato Beetle, Leptinotarsa decemlineata (Coleoptera: Chrysomelidae) evoked by mechanical and optical stimuli. Eur. J. Entomol. 99: 215-219.

Mill P. J. 1985: Structure and physiology of respiratory system. In Kerkut G.A. \& Gilbert L.J. (eds): Comprehensive Insect Physiology, Biochemistry and Pharmacology. Pergamon Press, Oxford, pp. 517-593.

Miller P.L. 1974: Respiration - aerial gas transport. In Rockstein M. (ed.): The Physiology of Insecta. Vol. 6. Academic Press, New York, San Francisco, London, pp. 345-402.

Miller P.L. 1981: Ventilation in active and inactive insects. In Herreid C.F. \& Fourtner C.R. (eds): Locomotion and Energetics in Arthropods. Plenum Press, New York, pp. 367-390.

SLÁMA K. 1976: Insect haemolymph pressure and its determination. Acta Entomol. Bohemoslov. 73: 65-75.

SLÁMA K. 1984: Recording of haemolymph pressure pulsations from the insect body surface. J. Comp. Physiol. 154: 635-643.

SlÁmA K. 1988: A new look at insect respiration. Biol. Bull. Mar. Biol. Lab. (Woods Hole) 175: 289-300.

SLÁmA K. 1999: Active regulation of insect respiration. Ann. Entomol. Soc. Am. 92: 916-929.

SLÁmA K. 2000: Extracardiac versus cardiac haemocoelic pulsation in pupae of the mealworm (Tenebrio molitor L.). J. Insect Physiol. 46: 977-992.

SlÁmA K. 2003: Mechanical aspects of heartbeat reversal in pupae of Manduca sexta. J. Insect Physiol. 49: 645-657.

SLÁMA K. \& FARKAŠ R. 2005: Heartbeat patterns during the postembryonic development of Drosophila melanogaster. $J$. Insect Physiol. 51: 489-503.

SLÁma K. \& NEVEN L. 2001: Active regulation of respiration and circulation in pupae of the codling moth (Cydia pomonella). J. Insect Physiol. 47: 1321-1336.

WASSERTHAL L.T. 1996: Interaction of circulation and tracheal ventilation in holometabolous insects. Adv. Insect Physiol. 26: 297-351.

Received June 8, 2005; revised and accepted November 8, 2005 